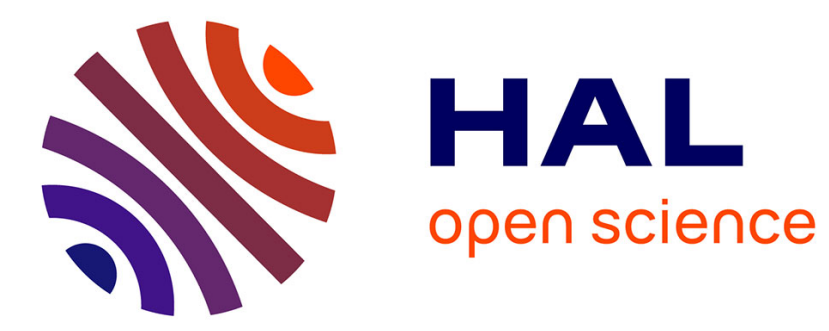

\title{
Binary systems of smectics A with enhanced nematic phase
}

\author{
R. Dabrowski, J. Szulc
}

\section{To cite this version:}

R. Dabrowski, J. Szulc. Binary systems of smectics A with enhanced nematic phase. Journal de Physique, 1984, 45 (7), pp.1213-1222. 10.1051/jphys:019840045070121300 . jpa-00209857

\section{HAL Id: jpa-00209857 https://hal.science/jpa-00209857}

Submitted on 1 Jan 1984

HAL is a multi-disciplinary open access archive for the deposit and dissemination of scientific research documents, whether they are published or not. The documents may come from teaching and research institutions in France or abroad, or from public or private research centers.
L'archive ouverte pluridisciplinaire HAL, est destinée au dépôt et à la diffusion de documents scientifiques de niveau recherche, publiés ou non, émanant des établissements d'enseignement et de recherche français ou étrangers, des laboratoires publics ou privés. 
Classification

Physics Abstracts

$61.30 \mathrm{E}-74.70 \mathrm{M}$

\title{
Binary systems of smectics A with enhanced nematic phase
}

\author{
R. Dąbrowski and J. Szulc \\ Military Technical Academy, 00-908 Warsaw, Poland \\ (Reçu le 10 novembre 1983, révisé le 8 février 1984, accepté le 28 février 1984)
}

\begin{abstract}
Résumé. - On a établi le diagramme de phases de mélanges binaires formés de smectiques $\mathrm{A}$ à couches monomoléculaires $(d \approx 1)$ et de smectiques $\mathrm{A}$ à couches bimoléculaires $(d>l)$. Trois types de diagramme de phases ont été trouvés pour les mélanges cités : i) La phase smectique n'est pas observée dans les gammes de concentration de 0,2-0,4 à 0,6-0,8 en fraction molaire. ii) On observe une réduction considérable de la stabilité de la phase smectique dans les limites de concentrations mentionnées ci-dessus. iii) La réduction de la stabilité de phase smectique n'est pas observée ou elle est tout à fait minime.

La séparation des régions correspondant aux phases smectiques A de types mono- et bimoléculaire par une phase nématique est liée à l'incommensurabilité des périodicités des couches smectiques. Il est suggéré que le mécanisme de cet effet est semblable à celui aboutissant à l'apparition de la phase nématique « réentrante ".
\end{abstract}

\begin{abstract}
Phase diagrams were determined for binary mixtures consisting of a monomolecular smectic $\mathrm{A}(d \approx l)$ and of a bimolecular one $(d>1)$. Three types of phase diagrams are observed : i) No smectic phase is observed in the concentration range of $0.2-0.4$ to $0.6-0.8$ mole fraction. ii) Only significant lowering of the phase stability is observed in the range specified in i). iii) No lowering of the smectic phase stability is observed or, if it occurs, it is very small. The observed nematic gap between the mono- and bimolecular $S_{A}$ phases is related to the incomensurate periodicities of the smectic layers. It is suggested that this phenomenon has a mechanism similar to that of the "re-entrant» nematic phase formation.
\end{abstract}

\section{Introduction.}

In many binary systems consisting of nematic compounds, smectic phase induction is observed when one of the compounds shows properties with electron acceptor features prevailing and the other one with electron donor features more pronounced. If one or both components have a smectic phase in the pure state, an extension of the range of existence and an increase of stability of the smectic phase in the mixture are observed. Many examples of pairs of compounds revealing such a behaviour have been described, e.g. by Domon and Billard [1], Engelen et al. [2], Griffin et al. [3].

An interesting phenomenon has been observed for several pairs of such compounds; the stability of the smectic phase increases with changing composition of the mixture in a continuous manner only on the side of one component, while on the side of the other component it decreases, and at a certain concentration of the components in the mixture the smectic phase disappears altogether. The region of existence of the enhanced smectic phase extends therefore only on the side of one component of the mixture and does not continue in the region where the smectic phase with the excess of the other component exists. Those smectic phases are separated by a nematic gap. Such properties are revealed by the system PCB-hexyloxyphenyl butylbenzoate described by $\mathrm{Oh}$ [4] as well as the systems consisting of esters and Schiff's bases described by Engelen $e t$ al. [5]. In the afore mentioned systems, one of the compounds yields bimolecular smectic A layers $(d>l)$ and the other compound yields monomolecular smectic A layers $(d \approx l)$. Their mixtures give an enhanced smectic with monomolecular layers [5] which joins in a continuous manner the smectic phase of the pure compound which has also a monomolecular layer.

A similar phenomenon of a strong enhancement of the nematic phase range at the cost of the smectic $A$ phase, which is reflected by the complete disappearance of the smectic phase in the range of concentrations close to equimolar proportions but without increase of the smectic phase stability in another 
concentration range, has been observed by the present authors in alkylcyclohexylbenzoic esters when attempts were made to identify their smectic phases by the miscibility method [6]. Such properties were revealed by mixtures composed of 4"-cyanophenyl 4-(trans-4'-ndecylcyclohexyl) benzoate (10CPCHB) and 4 "-isothiocyanatophenyl or 4"-acetoxyphenyl 4-(trans-4'-npentylcyclohexyl) benzoate (5TPCHB and 5APCHB, respectively). In order to better understand that phenomenon we measured the smectic layer spacing for those esters. It was observed that esters with groups $-\mathrm{NO}_{2}$ and $-\mathrm{CN}$, as previously found for many other compounds with such groups [7-9], have a smectic layer spacing greater than the length of the molecule, whereas the esters with the groups -NCS and -I have smectic layer spacings approximately equal to the length of the single molecule [10]. The dipole moments of the - $\mathrm{CN}$, - $\mathrm{NO}_{2},-\mathrm{NCS}$, -I terminal groups at the benzene ring are : 4.05, 4.01, 2.9 and 1.4 D, respectively [11]. As can be seen, the -NCS group is relatively less polar than the $-\mathrm{CN}$ or $-\mathrm{NO}_{2}$ groups but more polar than the -I group. Hence monomolecular smectic layers are produced by compounds not only with nonpolar terminal substituents but also with polar ones. Seurin et al. [12] made a similar finding earlier that alkoxybenzylidenehalogenoanilines also yield smectic monolayers.

The alkylcyclohexylbenzoic esters with -NCS and $-\mathrm{I}$, as well as with $-\mathrm{CN}$ and $-\mathrm{NO}_{2}$ terminal groups studied by us have smectic A phases. This seems to indicate that some binary systems composed of compounds that are smectics $\mathrm{A}$ of $\mathrm{A}_{1}$ type (with a monolayer, $d \approx l$ ) and of $\mathrm{A}_{\mathrm{d}}$ type (with a bilayer, $d>l$ ) are not compatible, and a change of concentration gives effects similar to those observed in systems revealing, with a change in temperature, the presence of re-entrant phases. Those phases have recently been the subject of great interest [13-16]. It would be interesting to know whether in both phenomena the cause of nematic phase formation is similar, and whether the mechanisms of the nematic phase formation differ or not. In order to acquire a better knowledge of the factors responsible for the nematic phase enhancement in systems consisting of compounds with smectic phases of $A_{d}$ and $A_{1}$ types, we tested a dozen or so binary systems composed of compounds with $-\mathrm{CN}$ and $-\mathrm{NO}_{2}$ as well as with -NCS and -I terminal groups. The results obtained relating to the phase diagrams of these systems are presented in this work.

\section{Experimental.}

The list of the compounds tested, their phase transition temperatures, the calculated lengths of the molecules as well as the measured smectic layer spacings are listed in table I. All the compounds were synthesized in our laboratory according to methods described previously [6] for compounds $1-10$ of the list, in [17] for compound 11, and in [18] for compound 12 .
The binary mixtures were prepared by weighing out on an analytical balance the pure compounds in adequate proportions in small glass crucibles. The total weight of the sample was $50 \mathrm{mg}$. In order to make homogenization of the sample easier, it was dissolved in chloroform prior to melting and the solvent was evaporated. The phase transition temperatures were measured using a VEB Analityk Dresden polarization microscope with a heated stage. In the vicinity of the phase transition temperatures the stage was heated at a rate of $0.5 \mathrm{~K} \mathrm{~min}^{-1}$. The measurements of the transition points $\mathrm{S} \rightarrow \mathrm{N}$ and $\mathrm{N} \rightarrow$ I were made in the heating and cooling cycles.

Usually those phase transitions took place in an interval narrower than $1 \mathrm{~K}$. However, if that interval was wider, the temperatures of the beginning and end of the transition range were marked on the diagram. The monotropic transitions were observed by placing on the stage, heated to a temperature close to the expected phase transition point, the samples heated by means of an additional heater to a temperature above the melting point.

\section{Results.}

3.1 BINARY SYSTEMS IN WHICH THE SMECTIC PHASE DISAPPEARS. - In figures $1 a-1 c$ phase diagrams are given of binary mixtures such as : 5 TPCHB-10CPCHB, 5TPCHB-10NPCHB, and 5TPCHB-9CPCHB. The three diagrams with all the others given in this work have been plotted in the temperature $\left({ }^{\circ} \mathrm{C}\right)$-composition (mole fraction) coordinate system. The experimental points representing phase transitions of the same type are marked in all of the diagrams in the same way. The three pairs of compounds to which figure 1 relates have phase diagrams of the same type. The solidmesophase interface reveals the presence of one simple eutectic. The nematic-isotropic liquid interface shows a small negative deviation from the value calculated according to the van Laar formula [19] describing the behaviour of a ideal solution :

$$
x_{A}^{\beta}=\frac{\exp \lambda_{A}^{\alpha \beta}\left(1-\exp \lambda_{B}^{\alpha \beta}\right)}{\exp \lambda_{A}^{\alpha \beta}-\exp \lambda_{B}^{\alpha \beta}}
$$

where :

$$
\begin{aligned}
\lambda_{\mathrm{A}}^{\alpha \beta} & =\frac{\Delta H_{\mathrm{A}(\alpha \rightarrow \beta)}}{R}\left(\frac{1}{T_{\mathrm{A}}}-\frac{1}{T}\right) \\
\lambda_{\mathrm{B}}^{\alpha \beta} & =\frac{\Delta H_{\mathrm{B}(\alpha \rightarrow \beta)}}{R}\left(\frac{1}{T_{\mathrm{B}}}-\frac{1}{T}\right) .
\end{aligned}
$$

The smectic-nematic phase transition is observed in a limited concentration range and does not even show an approximate agreement with equation (1). The concentration ranges in which the smectic phase exists are limited only to the intervals determined by the mole fractions : $0-0.2$ to 0.4 and $0.6-0.8$ to 1 . For concentrations between the given intervals the smectic phase is not observed even if the system is supercooled by several dozen degrees below the melting point. 
Table I. - The structural formulae, phase transition temperatures, molecular lengths $(l)$ and smectic layer spacings (d) for the compounds used for preparing the binary mixtures.

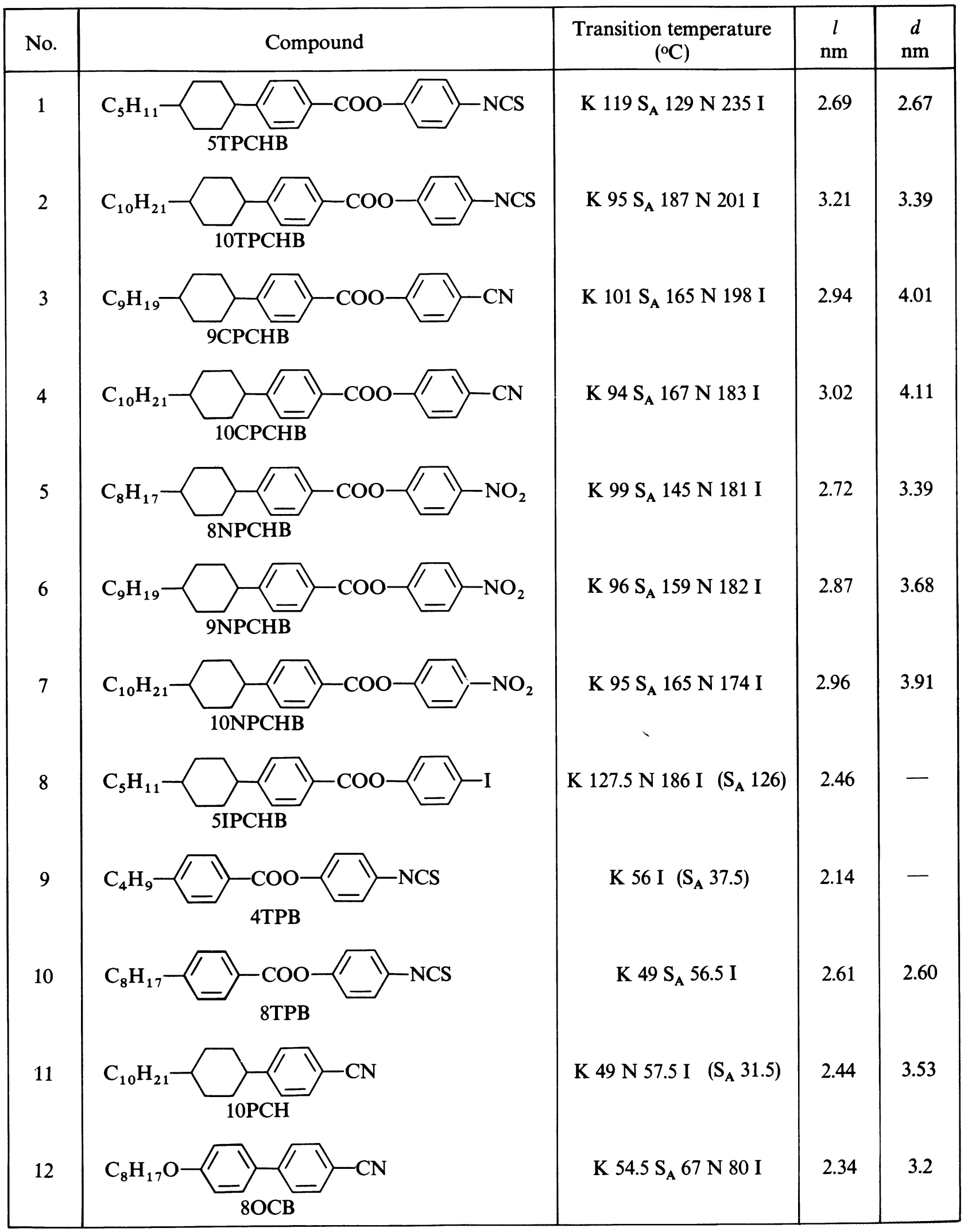



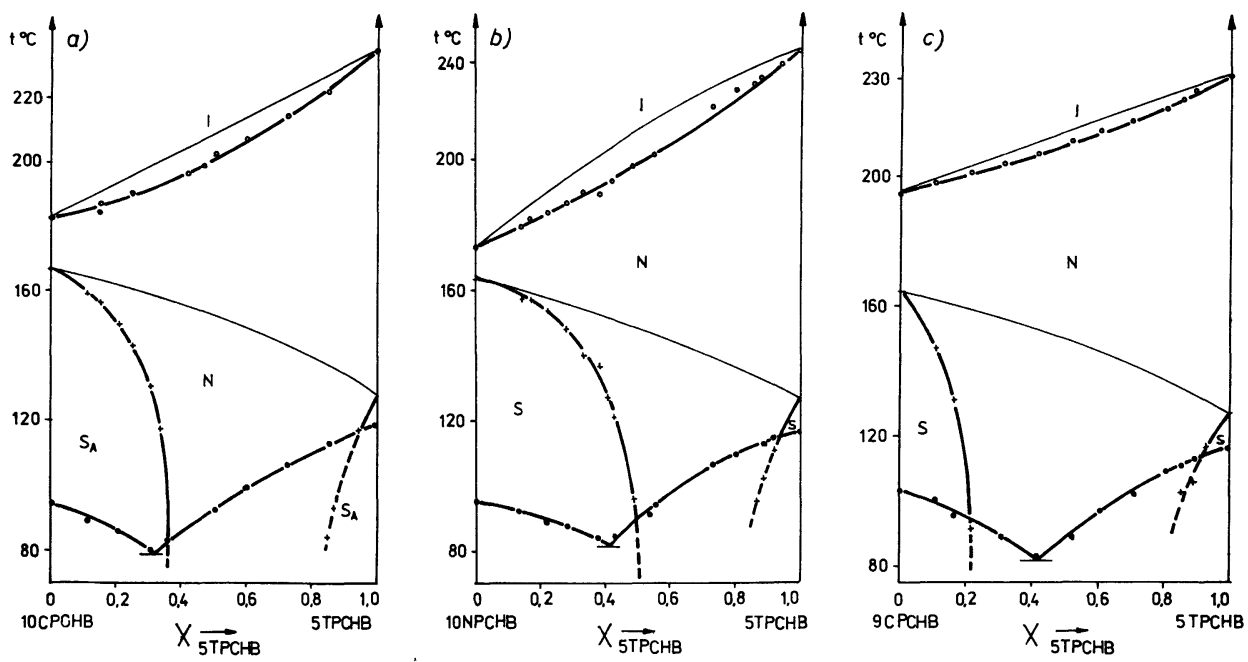

Fig. 1. - Phase diagrams of binary systems composed of isothiocyanatophenyl and cyanophenyl or nitrophenyl alkylcyclohexylbenzoates in which the decay of the smectic phase is observed.

The phase transitions are marked : - solid-mesophase/end melting point/, + smectic-nematic, $O$ nematic-isotropic liquid. Thin lines denote the theoretical boundaries of the phases $\mathrm{N} \rightarrow \mathrm{I}$ and $\mathrm{S} \rightarrow \mathrm{N}$ as calculated from eq. (1). The dashed line connects experimental points observed in supercooling state.

In the middle concentration range of those compounds in the mixtures, only the nematic phase exists, despite the fact that the individual compounds have a smectic phase. The temperature range in which the stable nematic phase is observed for such mixtures is sometimes several degrees wider than that observed for the pure components. The width of the nematic gap separating the range of existence of the smectic phase with the excess of one compound from that of the existence of the smectic phase with the excess of the other compound depends on the properties of the compounds present in the binary systems. It is found that the width of nematic gap is greater for compounds with the $-\mathrm{CN}$ terminal substituent than for those with the $-\mathrm{NO}_{2}$ group if the length of the alkyl substituent in both compounds is the same. This is in accordance with the generally known greater smectogeneity of the compounds with the $-\mathrm{NO}_{2}$ group as compared with those with the $-\mathrm{CN}$ group. A confirmation can be found, in the greater value of the phase transition temperature ratio, $T_{\mathrm{S} \rightarrow \mathrm{N}} / T_{\mathrm{N} \rightarrow \mathrm{I}}$, observed for nitro compounds as compared with cyano derivatives of the same molecular structure. These ratios are for the nitro esters $5,6,7$ tested by us : $0.8,0.87$ and 0.93 , respectively, while for analogous esters with cyano group they are lower, and amount to : $0.73,0.83$ and 0.91 . The widest concentration range $(0.22-0.78)$ that limits the existence of the smectic phase was observed within the scope of the tested binary systems for the paris of compounds selected so that they have alkyl substituents with 5 and 9 carbon atoms : 9CPCHB5TPCHB (Fig. 1c) and 9NPCHB-5TPCHB. For the pairs of compounds presented in figure 1 , as well as for others described later in this paper, it has been found that the $\mathrm{N} \rightarrow \mathrm{S}$ phase transition temperature decreases rapidly as the concentration of the second component increases in the mixture and finally that the decrease is so quick that the interface separating the nematic from the smectic phase is almost a vertical line. Thus for every pair of such compounds there are two limiting concentrations between which the system does not show smectogenic properties.

Phase diagrams of similar character have been observed for mixtures in which compound 5TPCHB was replaced by the isomorphic compound 5IPCHB (Fig. 2). The latter compound (iodophenyl ester) is like the isothiocyanate (5TPCHB) a smectic of $A_{1}$ type with a very similar stability of the smectic phase. In the case of such pairs as IIPCHB-10CPCHB and 5IPCHB-10NPCHB the solid curve on the phase
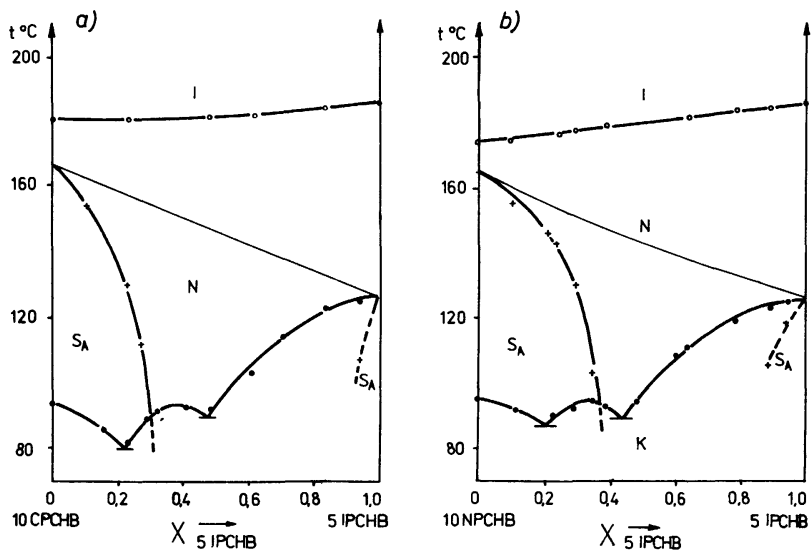

Fig. 2. - Phase diagrams of binary systems composed of iodophenyl and cyanophenyl or nitrophenyl alkylcyclohexylbenzoates in which the decay of the smectic phase is observed. 
diagram (Fig. 2) reveals the presence of one maximum and two eutectic points. This points to the formation of a molecular compound in a 2:1 molar ratio of $10 \mathrm{CPCHB}$ or 10NPCHB to 5IPCHB in the solid. This molar ratio may indicate that the dimeric structure characteristic of pure 10CPCHB and 10NPCHB in the mesogenic state is also preserved in the mixture with 5IPCHB in solid state. In the case of these esters and of similar compounds with the terminal groups $-\mathrm{CN},-\mathrm{NO}_{2}$, and $-\mathrm{CHO}$, the molecules are arranged in antiparallel fashion in the dimer. This is confirmed by the results of dielectric measurements. Considering the length and confirmation of the molecules as well as the spacing of the smectic layers, we proposed the arrangement of molecules in the dimers composed of the molecules of the tested esters [10] shown in figure 3. In the proposed structure, not only the aromatic rings but practically the whole rigid cores of the molecules superimpose. This may lead to the conclusion that entropic factors contribute largely to the stabilization energy. It is probable that in the nematic phase of those mixtures, the dimeric structure of 10CPCHB and $10 \mathrm{NPCHB}$ is also preserved, since the clearing temperature composition curves are consistent with the theoretical equation (1). The perfect solution equation is fulfilled here better than in the cases of the remaining pairs of compounds. This allows us to conclude that in the nematic phase of the mixtures of those compounds, no change in the character of the intermolecular interactions takes place as compared with those of the pure compounds.

A complete decay of the smectic phase in the middle concentration range was also observed for the mixtures : 10PCH-8TPB, 8OCB-4TPB and 10CPCHB8TPB (Figs. 4a-4c).

The polar terminal groups in these three pairs of compounds are the same as in the pairs of compounds whose diagrams are shown in figure 1 , but differences in shapes of molecules occur, which relate to a greater extent to their rigid cores than to the length of the alkyl groups.

The rigid elements of the molecules of the 10CPCHB8TPB pair of compounds whose diagram is shown in figure $4 \mathrm{c}$ have different lengths - two and three rings, respectively. This results in large differences in the clearing temperatures of the pure compounds. Therefore, the beginning and end of the $\mathrm{N} \rightarrow \mathrm{I}$ phase transition are more separated than for the remaining mixtures tested in this work, for which the $\mathrm{N} \rightarrow \mathrm{I}$

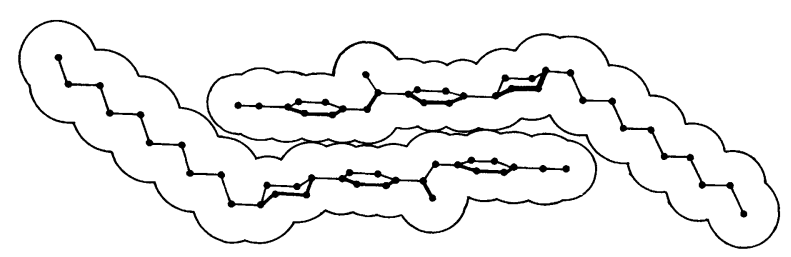

Fig. 3. - Model of molecular pairs occurring in 4"-cyanophenyl 4-(trans-4'-n-alkylcyclohexyl) benzoates.
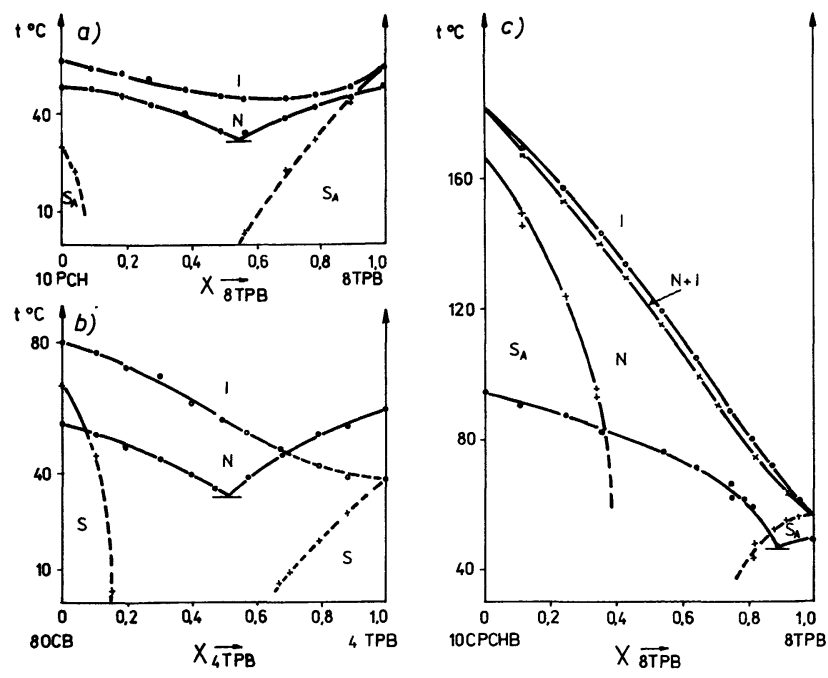

- Fig. 4. - Phase diagrams of binary systems composed of isothiocyanatophenyl alkylbenzoates and different cyano derivative in which the decay of the smectic phase is observed. $x$ : beginning of clearing.

transition interval observed is narrow - usually less than $1 \mathrm{~K}$. The 10PCH-8TPB pair consists of molecules with very similar total lengths, but their rigid cores differ in shape. This system is characterized by a single eutectic point. The clearing temperatures reveal strong negative deviations with a minimum on the side of isothiocyanate excess, approximately at the 8TPB : $10 \mathrm{PCH}$ ratio of $2: 1$. If it were assumed, by analogy to the formation of complexes leading to increased stability of the nematic phase in mixtures with smectic phase induction [20], that the observed lowering of the nematic phase stability is largely due to the splitting of $10 \mathrm{PCH}$ molecule dimers, then we could conclude on the basis of the shape of the clearing curve that there are no more dimers of $10 \mathrm{PCH}$ molecules when the system is rich in 8TPB. In the last binary system of this series (8OCB-4TPB, see Fig. 4c) the molecules differ to a lesser degree as regards shape, since both molecules there are aromatic, though different, rings. In this case the clearing temperature curve reveals much smaller negative deviations, and also with a minimum on the side of isothiocyanate excess.

\subsection{BINARY SYSTEMS IN WHICH A STRONG LOWERING OF} THE SMECTIC PHASE STABILITY IS OBSERVED. - In the tested binary mixtures we found three pairs of compounds that reveal a fairly high lowering of the smectic phase stability, but the smectic phase of one component joins the smectic phase of the other one in a continuous way (see Figs. $5 \mathrm{a}-5 \mathrm{c}$ ), these are : 5TPCHB8NPCHB, 8TPB-8OCB and 10TPCHB-5IPCHB. The first two pairs consist of compounds that are smectic $A$ with different structures of the smectic layers (monoand bilayers). The third pair consists of compounds which each yield a smectic A a monolayer structuring. The first two pairs (Figs. 5a and 5b) have the smectic phase stability minimum close to the equimolar range. 

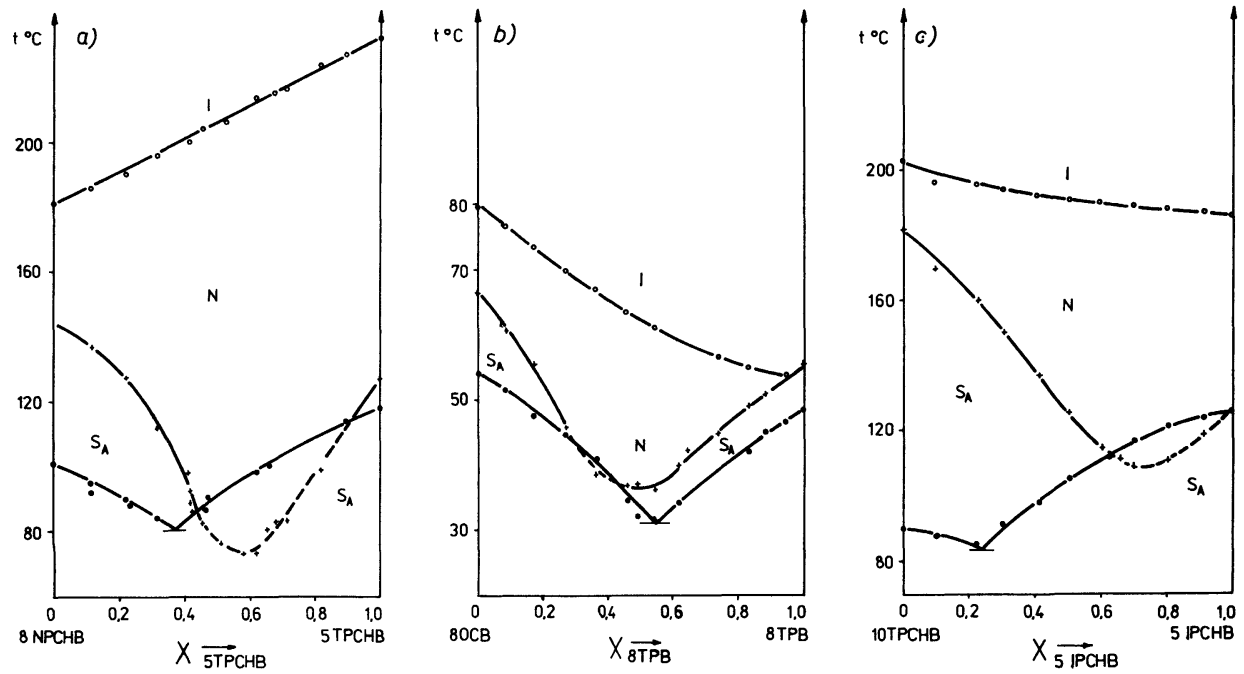

Fig. 5. - Phase diagrams of binary systems in which a significant lowering of the smectic phase stability is observed : (a) and (b) mixture of compounds yielding smectic mono- and bilayers, (c) mixture of compounds yielding smectic monolayers.

The minimum of the third pair is shifted towards the iodoester excess (Fig. 5c). The clearing temperatures of the 8NPCHB-5TPCHB system varies linearly with temperature. In this case the molecules forming this pair have almost identical lengths (see Table I). The clearing temperature curve of the 8OCB-8TPB system is concave with a minimum at the triple point of coexistence of the $\mathrm{N}, \mathrm{S}_{\mathrm{A}}$ and isotropic phases.

3. 3 SYSTEMS IN WHICH NO LOWERING OF THE SMECTIC PHASE STABILITY IS OBSERVED OR THAT LOWERING IS SMALL. - The lack of greater changes in the smectic phase stability was observed in systems formed of compounds that possess smectic layers of the same kind - bimolecular as in figure $6 \mathrm{a}$ or monomolecular as in figures 7a-7c, but also in those composed of compounds with various layer structures of the smectic phase (Figs. 6b-6d). Hence, the strong lowering of the smectic phase stability does not result from the fact that the compound making up the mixture have different structuring of the smectic A layer, but is due to other factors. Let us analyse the diagrams shown in figures 6 and 7 and the properties of the relevant molecules. The phase diagram of the 10CPCHB-10NPCHB system (Fig. 6a) shows that $T_{\mathrm{N} \rightarrow 1}=f(x)$ and $T_{\mathrm{S}_{\mathrm{A}} \rightarrow \mathrm{N}}=f(x)$ varies linearly, while the $T_{\mathrm{K} \rightarrow \mathrm{S}_{\mathrm{A}}}=f(x)$ curve is characteristic of a system yielding solid solutions of unlimited solubility with a minimum in the solidus. The compounds forming that pair have almost identical properties; the same total length of the molecule and alkyl substituents of equal length, the same shape and almost the same spacing of the smectic layer of $A_{d}$ type. If we consider all these factors, the observed character of the phase diagram becames obvious. The character of the phase diagram of the pairs of compounds 10NPCHB-10TPCHB and $10 \mathrm{CPCHB}-10 \mathrm{TPCHB}$ is very similar (Figs. $6 \mathrm{~b}$ and $6 \mathrm{c}$ ).
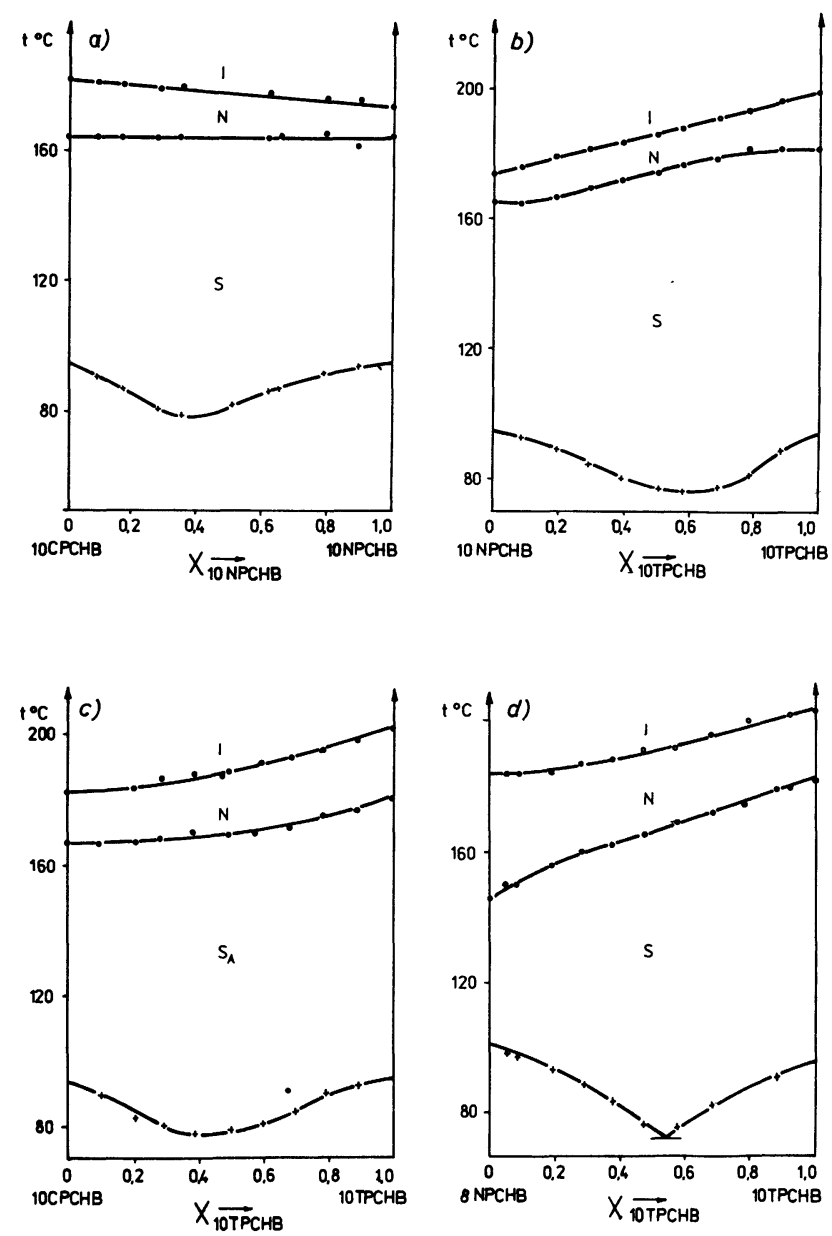

Fig. 6. - Phase diagrams of binary mixtures in which $T_{\mathrm{N} \rightarrow \mathrm{S}}$ varies with composition approximately as in a perfect solution : (a) mixture of two smectics with bimolecular layers, (b), (c) and (d) mixtures of smectics with mono- and bimolecular layers. 

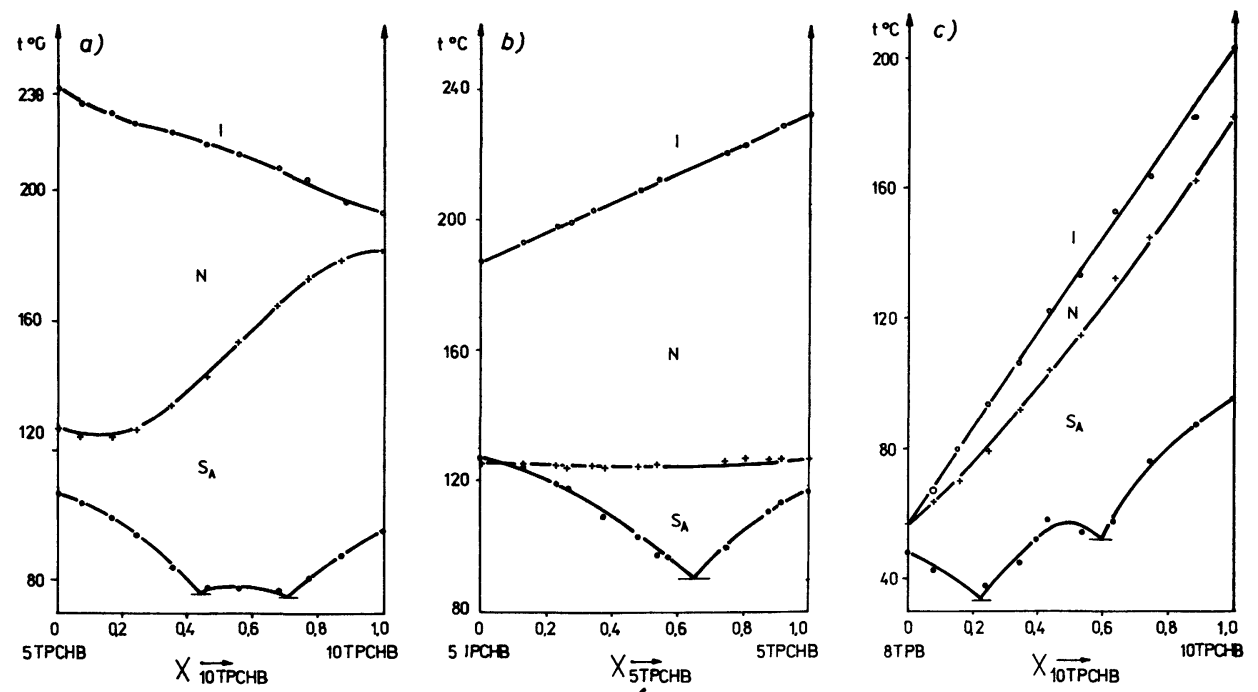

Fig. 7. - Phase diagrams of systems composed of two smectics with monolayers whose phase boundaries $\mathrm{N} \rightarrow \mathrm{S}$ and $\mathrm{N} \rightarrow \mathrm{I}$ reveal small deviations from the perfect solution or vary like those of perfect solutions.

In these pairs of compounds the single molecules are of similar shape and length, but their smectic layers are of different character (types $A_{1}$ and $A_{d}$ ) and differ moderately regarding spacing. This smectic layer incommensurate periodicity is revealed only by small deviations of $T_{\mathrm{S} \rightarrow \mathrm{N}}$ from linearity. The $\mathrm{N} \rightarrow \mathrm{S}$ interface is concave for 10NPCHB-10TPCHB on the side of 10NPCHB excess, and convex on the side of 10TPCHB whereas for 10CPCHB-10TPCHB it is only concave. For the pair of compounds 8NPCHB-10TPCHB (Fig. 6d) the $\mathrm{N} \rightarrow \mathrm{S}$ interface observed is convex and the $\mathrm{N} \rightarrow \mathrm{I}$ interface is concave. The differences in the lengths of the molecules in the last pair are quite significant, while the spacings of the smectic layers are almost identical. The differentiation of the molecular dimension is big enough to produce a lowering of their mutual solubility in the solid and therefore we observe a single eutectic point in the solidus. That differentiation does not produce, however, a disturbance of the smectic phase structure owing to the similarity of the smectic layer spacings.

Figures $7 \mathrm{a}-7 \mathrm{c}$ present diagrams of the following pairs of compounds : 10TPCHB-5TPCHB, 10TPCHB8TPB and 5TPCHB-5IPCHB, which all give $A_{1}$ smectic layers.

The first two pairs consist of isothiocyanates differing in molecular length. Their solidus points to the formation of a molecular compound of low stability in the solid phase at the 1:1 molar ratio. The $T_{\mathrm{S} \rightarrow \mathrm{N}}=f(x)$ interfaces show small deviations from linearity, which in the case of the first pair are both positive and negative and greater than for the second pair where only negative deviations are observed.

The third pair (Fig. 7b) consist of molecules of the same total length and with the same alkyl substituent but differing largely as regards the shape of the second terminal substituent (-I) and (-NCS). This pair presents a classical system in which there are no specific interactions in the solid, and for smectic and nematic phases - the $T_{\mathrm{N} \rightarrow \mathrm{I}}=f(x), \quad T_{\mathrm{S} \rightarrow \mathrm{N}}=f(x)$ and $T_{\mathrm{K} \rightarrow \mathrm{S}}=f(x)$ interfaces are in agreement with equation (1).

\section{Discussion.}

In the tested binary mixtures, composed of compounds that have a $S_{A}$ phase and are selected so that one component gives only bimolecular smectic phases and the other monomolecular ones, three kinds of behaviour are observed for which three types of phase diagrams correspond :

i) The $S_{A}$ phases are incompatible and the smectic phase disappears (is destroyed) in the concentration range of $0.2-0.8$.

ii) The smectic phase exists in the whole concentration range but its stability is strongly lowered close to the equimolar concentration range.

iii) The smectic phase stability is not lowered or lowered only slightly. The mixture reveals properties similar to those of a perfect solution.

Such a differentiated behaviour leads us to the conclusion that separation of the smectic phases of both components by a nematic phase gap is indeed observed when bimolecular and monomolecular smectic A layers are in contact, however, this condition is insufficient. To find the real cause responsible for the behaviour of certain pairs of compounds we compared the ratios of molecule lenghts (Table II) and the smectic layer spacings for all of the tested pairs of compounds. The disappearance of the smectic phase was observed in those mixtures (pairs 1-9) in which the ratio of molecule lengths $l / l^{\prime}$ varies in the range 1.071.28 , and the ratio of smectic layer spacings varies 
Table II. - Comparison of the molecular length ratios $\left(l / l^{\prime}\right)$ and smectic layer spacings $\left(d / d^{\prime}\right)$ for compounds making up the tested binary mixtures.

\begin{tabular}{|c|c|l|l|}
\hline No. & Binary system & $l / l^{\prime}$ & $d / d^{\prime}$ \\
\hline 1 & 10CPCHB-5TPCHB & 1.13 & 1.54 \\
2 & 10NPCHB-5TPCHB & 1.10 & 1.46 \\
3 & 9CPCHB-5TPCHB & 1.09 & 1.50 \\
4 & 9NPCHB-5TPCHB & 1.07 & 1.38 \\
5 & 10СРCHB-5IPCHB & 1.23 & $1.67\left(^{*}\right)$ \\
6 & 10NPCHB-5IPCHB & 1.20 & $1.59\left(^{*}\right)$ \\
7 & 10 CPCHB-8TPB & 1.16 & $1.58\left(^{*}\right)$ \\
8 & 10РCH-8TPB & 1.07 & 1.36 \\
9 & 8ОСВ-4TPB & 1.09 & $1.50\left(^{*}\right)$ \\
\hline 10 & 10TPCHB-4TPB & 1.50 & $1.58\left(^{*}\right)$ \\
\hline 11 & 8NPCHB-5TPCHB & 1.01 & 1.27 \\
12 & 8OCB-8TPE & 0.89 & 1.23 \\
13 & 10TPCHB-5IPCHB & 1.30 & $1.38\left(^{*}\right)$ \\
\hline 14 & 10TPCHB-5TPCHB & 1.19 & 1.27 \\
15 & 10TPCHB-8TPB & 1.23 & 1.30 \\
16 & 10СРCHB-10TPCHB & 0.94 & 1.21 \\
\hline 17 & 5TPCHB-5IPCHB & 1.09 & $1.09\left(^{*}\right)$ \\
18 & 10CPCHB-10NPCHB & 1.02 & 1.05 \\
19 & 10NPCHB-10TPCHB & 0.92 & 1.15 \\
20 & 8NPCHB-10TPCHB & 0.85 & 1.00 \\
\hline
\end{tabular}

$\left(^{*}\right)$ The spacings of the 4TPB and 5IPCHB smectic layers have been assumed to be equal to the lengths of the molecules by analogy to 8 TPB and 10IPCHB, for which such a correlation was found to occur [23].

from 1.36 to 1.67 . For the remaining cases the $l / l^{\prime}$ ratio varied within similar, fairly wide limits of 0.85-1.30. while the $d / d^{\prime}$ ratio was smaller decreases as the properties of the system for $\mathrm{S}_{\mathrm{A}} \rightarrow \mathrm{N}$ transition approach those of a perfect solution. For the particular types of phase diagram the ratio $d / d^{\prime}$ of smectic layer spacings has the following values :

1.36-1.67: when disappearance of the smectic phase in observed ;

1.23-1.27 : a significant lowering of the smectic phase stability occurs;

1.21 : small lowering of the smectic phase stability is observed;

1.00-1.15 : no lowering of the smectic phase stability occurs.

It follows from this list that the ratio of the smectic phase spacings of the pure compounds, that should be greater than 1.3, is the main factor responsible for the decay of the smectic phase. The magnitude of the nematic gap observed between the $S_{A}$ smectic phases is also affected by other factors due to differences in the chemical and geometric structures of the compounds making up the binary mixture.
It would be interesting to know whether the decay of the smectic phase is observed only in mixtures composed of smectics with mono- and bimolecular layers or whether it is independent of such a combination of compounds, and will be always observed when the smectic layers achieve sufficiently high incommensurate periodicities. From among the investigated compounds only one pair can be tested; 10TPCHB-4TPB, where both components are $A_{1}$ type smectics and their $d / d^{\prime}$ ratio is sufficiently high, amounting to about 1.58 . One component of that system has a monotropic smectic phase and the $\mathrm{S} \rightarrow \mathrm{N}$ transition is almost $20 \mathrm{~K}$ below the melting point. In the considered mixture of compounds we observe a very strong lowering of the smectic phase stability (Fig. 8), however, because of difficulties in achieving deep supercooling, we cannot claim with full conviction that the smectic phases are separated by a nematic gap. In order to solve that problem, a pair of compounds having more conveniently distributed smectic ranges should be selected. Nevertheless, in the light of the obtained results one can state with full conviction that in a mixture composed of mono- and bilayer smectics the lowering of the smectic phase stability is always greater than in a mixture composed of two smectics with layers of the same kind, the layer spacing ratio being the same in both cases. We can find support for this opinion if we compare the diagrams binary systems Nos. 11 and 12 with Nos. 13 and 14 (numbering according to Table II); see figures $5 \mathrm{a}-5 \mathrm{c}$ and $7 \mathrm{a}$. The ratios of the smectic layer spacings are for all four pairs of compounds are very similar, but for the two first pairs the observed lowering of the smectic phase stability is much greater than for the remaining two.

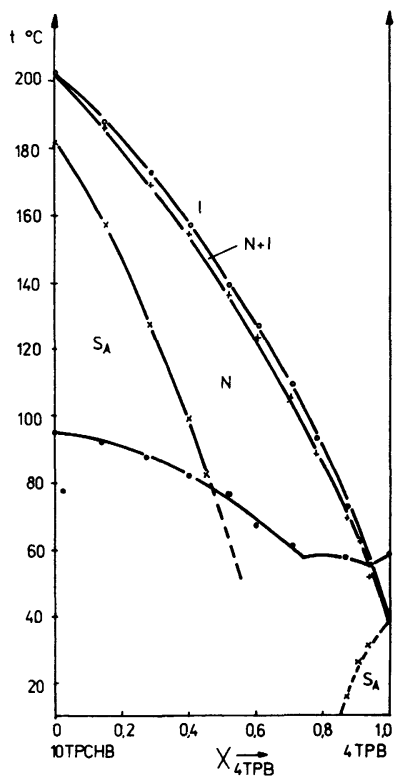

Fig. 8. - Phase diagram of a system composed of two smectics A yielding monolayer spacing ratio being 1.58 . 
What takes place in the smectic layer of a mixture composed of smectics differing significantly as regards the spacing of the smectic layers, especially if the intermolecular forces in the smectic layers made up of the pure components are greater than in their mixtures? This situation does not favour the exchange of molecules in layers made up of different molecules, and therefore does not lead to the averaging of the layer spacing; more probably bimolecular or greater groups composed of molecules of the same kind exists next to each other. Hence the smectic layer of the excess component have strong defects (of vacancy type) and their number will increase with the growing concentration of the second component and will reach a maximum for the equimolar composition. At the same time the packing density of the mixture should decrease, since ever greater spaces will be vacant.

A loose filling of space will favour weakened molecular interactions in the smectic phase and enhance greater mobility of the molecules in the smectic phase and their interlayer exchange.

When a certain limiting concentration of defects is exceeded, the smectic phase can no longer exist since its density is too small to provide sufficiently high attractive forces in the smectic layers that would be capable of inhibiting the growing mobility of the molecules. The nematic phase must be formed since it ensures a better packing of the mixture than the smectic phase. The explanation presented here of the disappearance of the smectic phases is only hypothetical and requires experimental verification.

It would be interesting therefore, to inspect the variation of the density as a function of composition at various temperatures for such mixtures. Let us pose the question : is the nematic phase really more densely packed than the smectic one in a certain concentration range ?

The observed singularities of mixtures consisting of smectics differing in layer spacing (especially of $A_{1}$ and $A_{d}$ types) reveal features very similar, in our opinion, to those observed in the re-entrant nematic phase; the only significant difference consists in that transition from the $A_{1}$ to the $A_{d}$ smectic region is not due to temperature, but is due to concentration changes. The nature of the nematic phase separating the smectic phases is in both cases identical : it is composed of incommensurate molecules or aggregates, some agregates being composed of single molecules and some dimers.

Hardouin et al. [21] found, by X-ray scattering, two types of cybotactic groups in the re-entrant nematic phase of 4'-(octyloxybenzoyloxy)-4-cyanostilbene (T8). Some of those groups are characteristic of the high-temperature $S_{A_{d}}$ phase and some of the low-temperature $S_{A_{1}}$ one. The nematic phase reveals strong pre-transition effects on the sides of both lower and higher temperatures.

The studies of Cladis [22] on the effect of pressure on $80 C B$ have shown that there exists a density interval optimal for the stabilization of the smectic layers. A too-low or too-high density of the system leads to the decay of the smectic phase due, in the first case to the decrease of the attractive forces in the layer, and in the second case to the increase of repelling forces.

In our opinion, further testing of the properties of mixtures composed of molecules that have polar terminal groups and are able to form smectic monoor bilayers in the pure state will permit a better knowledge of incommensurate systems as well as of the re-entrant nematic phase. It seems that density measurements as well as dielectric and X-ray studies are particularly useful for obtaining additional information facilitating the interpretation of the observed features of the phase diagrams. We intend therefore to continue in the near-future our studies along that line.

References

[1] Domon, M., Billard, J., J. Physique Colloq. 40 (1979) C3-43.

[2] Engelen, B., Schneider, F., Z. Naturforsch. 33a (1978) 1077.

[3] Griffin, A. C., Buckley, N. W., Havens, S. J., The Physics and Chemistry of Liquid Crystal Devices, Sprokel, G. J., ed. (Plenum Publ. Corp., New York) 1980 , p. 305.

[4] Он, C. S., Mol. Cryst. Liq. Cryst. 42 (1977) 1.

[5] Engelen, B., Heppke, G., Hopf, R., Schneider, F., Mol. Cryst. Liq. Cryst. Lett. 49 (1979) 193.

[6] Dabrowski, R., Dziaduszek, J., Szczuciński, T., Biul. WAT. 32 no 2 (1983) 3.

[7] Brownsey, G. J., Leadbetter, A. J., Phys. Rev. Lett. 44 (1980) 1608.
[8] Cladis, P. E., Guillon, D., Daniels, W. B., Griffin, A. C., Mol. Cryst. Liq. Cryst. Lett. 56 (1979) 89.

[9] Hardouin, F., Levelut, A. M., Benattar, J. J., Sigaud, G., Solid State Commun. 33 (1980) 337.

[10] Dabrowski, R., Baran, J., Przedmojski, J., Pura, B., Biul. WAT 32 no 8 (1983).

[11] Minkin, V. I., Osipov, O. A., Zhdanov, Yu. A., Dipolnye Momenty v Organicheskoi Khimii, Khimiya 1968.

[12] Seurin, P., Guillon, D., Skoulies, A., Liquid Crystals of One- and Two-Dimensional Order, Helfrich, W., Heppke, G., eds. (Springer Verlag, Berlin) 1980. p. 146.

[13] Guillon, D., Cladis, P. E., Stomateff, J., Phys. Rev. Lett. 41 (1978) 1598. 
[14] Hardouin, F., Sigaud, G., Achard, M. F., GaspaRoux, H., Phys. Lett. 71A (1979) 347.

[15] Demus, D., Pelzl, G., Wiegeleben, A., Weissflog, W., Mol. Cryst. Liq. Cryst. Lett. 56 (1980) 289.

[16] Prost, J., Liquid Crystals of One- and Two-Dimensional Order, Helfrich, W., Heppke, G., eds. (SpringerVerlag, Berlin) 1980, p. 125.

[17] Dabrowski, R., Szczuciński, T., Mol. Cryst. Liq. Cryst. 88 (1982) 55.

[18] Gray, G. W., Harrison, K. J., Nash, J. A., Coustant, J., Hulme, D. S., Kirton, J., Raynes, E. P., Proceedings of 166th National A.C.S. Meeting on Ordered Fluids and Liquid Crystals, Chicago, August 1973, Porter, R. S., Johnson, J. F., eds.
(Plenum Press, New York-London) 1974, vol. 2 , p. 617.

[19] Domon; M., Billard, J., Pramana Suppl. no 1 (1975) 131.

[20] Waclawek, W., Dąbrowski, R., Domagala, A., Mol. Cryst. Liq. Cryst. 84 (1982) 255.

[21] Hardouin, F., Levelut, A. M., Benattar, J. J., Sigaud, G., Liquid Crystals of One- and TwoDimensional Order, Helfich, W., Heppke, G., eds. (Springer-Verlag, Berlin) 1980, p. 147.

[22] Cladis, P. E., Bogardus, R. K., Aadsen, D., Phys. Rev. 18A (1978) 2292.

[23] Dabrowski, R., Przedmojski, J., Baran, J., Crystal Res. Technol. (in press). 